\title{
Intermolecular Resonance Correlates Electron Pairs Down a Supermolecular Chain: Antiferromagnetism in K-Doped p-Terphenyl
}

\author{
Natalia A. Gadjieva, ${ }^{\dagger}$ Péter Szimai, ${ }^{\beta}$ Olivér Sági, ${ }^{\beta}$ Pere Alemany, ${ }^{\dagger}$ Sergio Conejeros, ${ }^{\S}$ Daniel W. Paley, ${ }^{\dagger}$ Raúl Her- \\ nández Sánchez, ${ }^{\diamond}$ Brandon Fowler, ${ }^{\dagger}$ Samuel R. Peurifoy, ${ }^{\dagger}$ Amymarie K. Bartholomew, ${ }^{\dagger}$ Bálint Náfrádi, ${ }^{\beta}$ László \\ Forró, ${ }^{\beta}$ Xavier S. Roy, ${ }^{\dagger}$ Patrick Batail, ${ }^{\square,{ }^{*}}$ Enric Canadell, ${ }^{\Delta^{*}}$ Michael L. Steigerwald, ${ }^{+^{*}}$ Colin Nuckolls ${ }^{+^{*}}$ \\ ${ }^{\dagger}$ Department of Chemistry, Columbia University, New York, New York 10027, United States \\ ${ }^{\beta}$ Laboratory of Complex Matter Physics, Ecole Polytechnique Fédérale de Lausanne 1015 Lausanne, Switzerland \\ ${ }^{\ddagger}$ Departament de Ciència de Materials i Química Física and Institut de Química Teòrica i Computacional (IQTCUB), Universitat de Barcelona, \\ Martí i Franquès 1, Barcelona 08028, Spain \\ ${ }^{\S}$ Departamento de Química, Universidad Católica del Norte, Av. Angamos 0610, Antofagasta 124000, Chile \\ ${ }^{\Delta}$ Institut de Ciència de Materials de Barcelona (ICMAB-CSIC), Campus de la UAB, 08193, Bellaterra, Spain \\ ${ }^{\diamond}$ Department of Chemistry, University of Pittsburgh, 219 Parkman Ave, Pittsburgh, PA 15260, United States
}

MOLTECH-Anjou, UMR 6200, CNRS, Universite d'Angers, 49045 Angers, France

\begin{abstract}
Recent interest in potassium doped p-terphenyl has been fueled by reports of superconductivity at $T_{c}$ values surprisingly high for organic compounds. Despite these interesting properties, studies of the structure-function relationships within these materials have been scarce. Here we isolate a phase-pure crystal of potassium doped p-terphenyl: $[\mathrm{K}(222)]_{2}\left[p\right.$-terphenyl $\left.{ }_{3}\right]$. Emerging antiferromagnetism in the anisotropic structure has been studied in depth by magnetometry and electron spin resonance. Combining these experimental results with density functional theory calculations, we describe the antiferromagnetic coupling in this system that occurs in all 3 crystallographic directions. The strongest coupling was found along the ends of the terphenyls, where the additional electron on neighboring $p$-terphenyls antiferromagnetically couple. This delocalized bonding interaction is reminiscent of the doubly degenerate resonance structure depiction of polyacetylene. These findings hint towards magnetic fluctuation-induced superconductivity in potassium-doped $p$-terphenyl, which has a close analogy with high $\mathrm{T}_{\mathrm{c}}$ cuprate superconductors.
\end{abstract}

Introduction: Doping of polycyclic aromatic hydrocarbons (PAHs) and fullerenes are known to produce unique emergent properties such as superconductivity, quantum spin liquids, Mott metal-insulator transitions, and antiferromagnetism. ${ }^{1-7}$ Recently, interest in alkali-doped organics has reemerged after reports of superconductivity with increasing $\mathrm{T}_{c}$ from $\mathrm{K}_{3}$ Phenanthrene $\left(\mathrm{T}_{\mathrm{c}}=5 \mathrm{~K}\right)^{8} \quad$ to $\mathrm{K}_{3.3}$ Picene $\quad\left(\mathrm{T}_{\mathrm{c}}=18 \mathrm{~K}\right),{ }^{9} \quad \mathrm{Cs}_{2} \mathrm{RbC}_{60} \quad\left(\mathrm{~T}_{\mathrm{c}}=33 \mathrm{~K}\right),{ }^{10}$ $\mathrm{K}_{3.17}$ Dibenzopentacene $\left(\mathrm{T}_{c}=33 \mathrm{~K}\right)^{11}$ and the most recent reports of superconductivity of K-doped $p$-terphenyl with an extremely high $\mathrm{T}_{\mathrm{c}}$ of $123 \mathrm{~K} .{ }^{12}$ In these systems, there is a dearth of structural data on the molecular packing and the arrangement of the dopant atoms. ${ }^{1,13,14}$ Furthermore, reproducibility of superconducting alkalidoped PAHs remains controversial, since no repeat experiments on superconductivity at $33 \mathrm{~K}$ have been reported. ${ }^{15}$ Thus an understanding of the structure/function relationship in potassium doped $p$-terphenyl is of importance to provide design rules for high temperature superconducting materials from PAHs. The superconducting fraction in the reports on superconductivity in $p$-terphenyl was found to be low, raising the intriguing possibility that reaction chemistry and crystallography can help understand this important system. Identifying the structure and electronic behavior of ensembles of $p$-terphenyl radical anions is essential to understanding the superconducting phase and its related properties.

Controlling the reactivity of alkali metals is inherently problematic and contributes to the difficulty of obtaining phase-pure materials. ${ }^{16}$ In order to elucidate the fundamental understanding of the alkali metal doped terphenyl we developed the new approach, presented here, based on the use of cryptands. We report a new phase-pure system: $[\mathrm{K}(\mathbf{2 2 2})]_{2}\left[p\right.$-terphenyl $\left.{ }_{3}\right]$ where the [2.2.2]Cryptand, (222), is a polydentate ligand that selectively encapsulates $\mathrm{K}^{+}$ions, and thus preventing the alkali metal from forming ion- $\pi$ interactions. Crystallography reveals a stoichiometry of three $p$-terphenyl molecules and two chelated $\mathrm{K}^{+}$ions. The crystal contains two different $p$-terphenyls; the two doped $p$-terphenyls align as $1 \mathrm{D}$ chains around a column of $\mathrm{K}(\mathbf{2 2 2})$. This chain undergoes weaker interactions all along the solid, resembling the establishment of incipient bonds. Magnetic resonance reveals that the system is antiferromagnetically (AFM) coupled and DFT calculations show that the coupling is the largest along the $p$-terphenyl chain.

The appearance of the AFM state in our material should be highlighted, since a proximity to superconductivity has been made in various systems, including cuprates, Bechgaard salts, and fullerides. ${ }^{17-19}$ Among the latter, $\mathrm{Cs}_{3} \mathrm{C}_{60}$, was found to undergo a transition from AFM insulating state to superconducting state at 38 $\mathrm{K}$, under pressure. Since an attractive interaction between electrons is a requirement to form Cooper pairs, electron-electron interaction emerging in superconductors must overcome the Coulomb repulsion. Although organic superconductors are generally classified as BCS type (Bardeen-Cooper-Schrieffer), the nodal $s$ - or $d$ wave pairing symmetry for the superconducting gap (if any) is largely an open question for PAHs due to the lack of structural data. 
a)

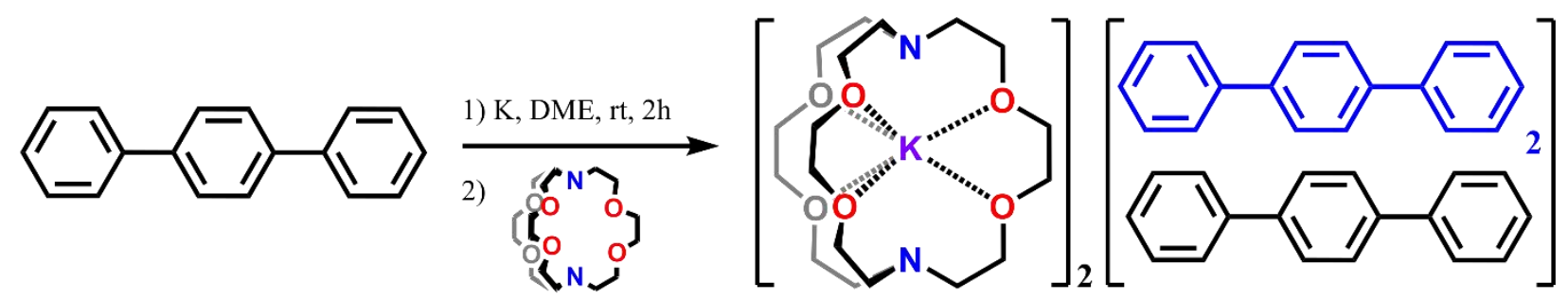

b)

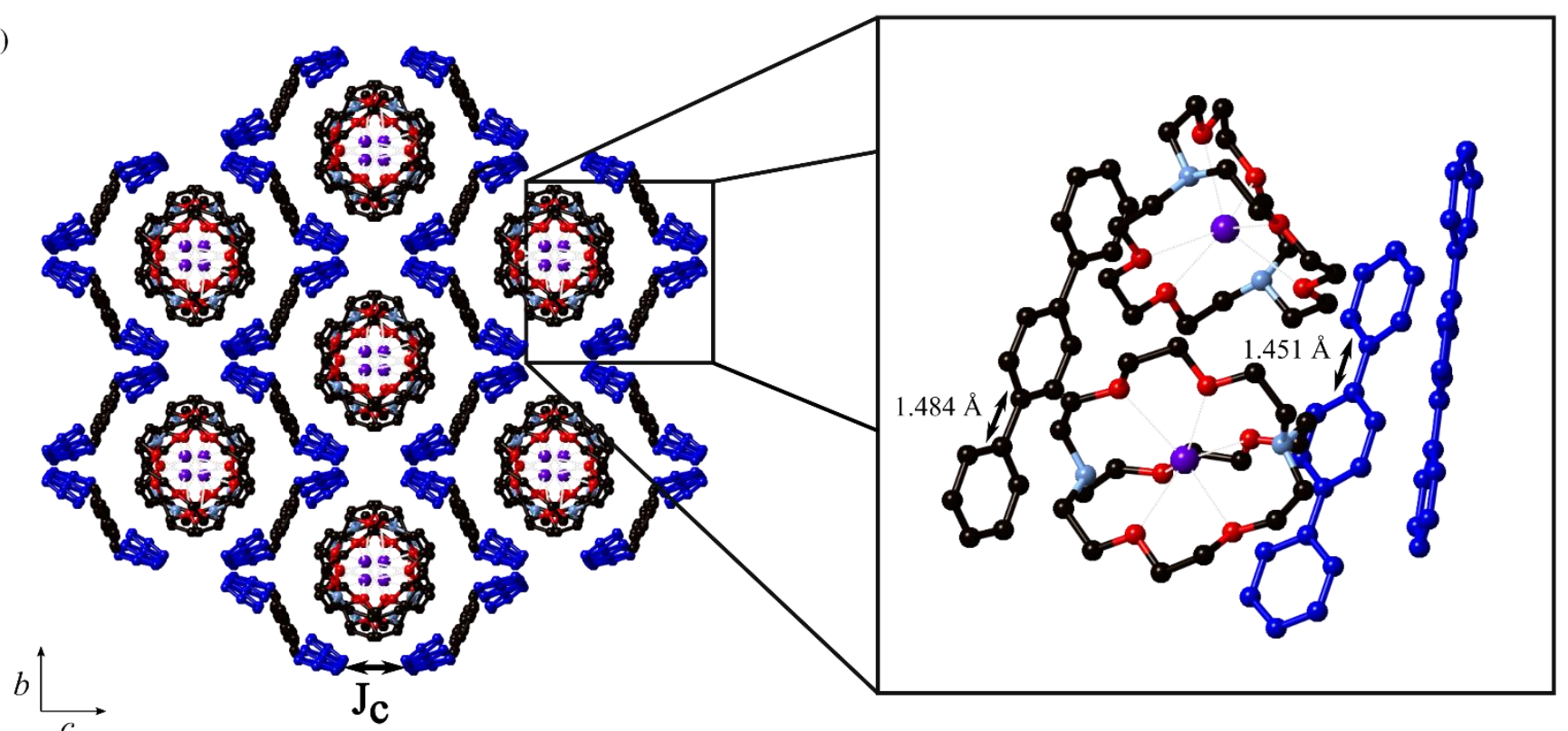

c)

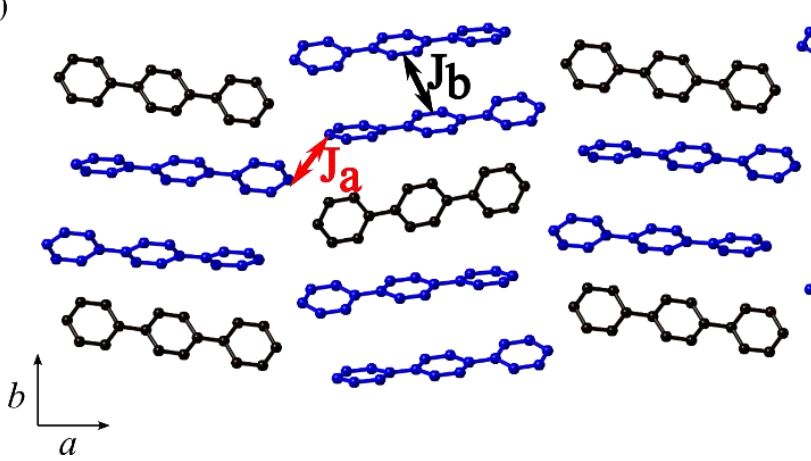

d)
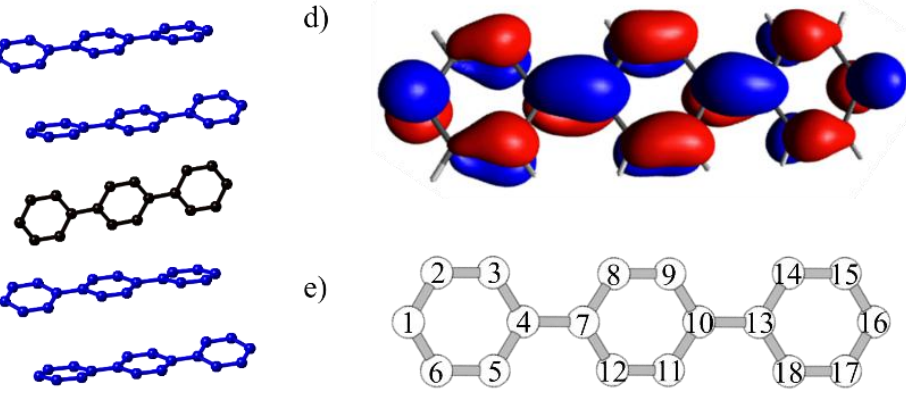

e)

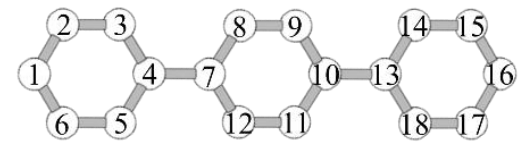

Figure 1: a) Synthesis of $[\mathrm{K}(\mathbf{2 2 2})]_{2}\left[p\right.$-terphenyl $\left.{ }_{3}\right]$ b) View of the $[\mathrm{K}(\mathbf{2 2 2})]_{2}\left[p\right.$-terphenyl $\left.{ }_{3}\right]$ structure along the $a$ axis with visible channels of (222) chelated to the $\mathrm{K}^{+}$ion, surrounded by $p$-terphenyl molecules. The insert shows a zoom-in on the unit cell, with two unique pterphenyls; containing neutral molecule (in black) and doped ones (in blue). Magnetic exchange coupling $J_{c}$ between doped $p$-terphenyls is depicted. c) View of $[\mathrm{K}(\mathbf{2 2 2})]_{2}\left[p\right.$-terphenyl $\left.{ }_{3}\right]$ along the $c$ axis $(\mathrm{K}(\mathbf{2 2 2})$ removed from the image). The structure shows $p$-terphenyl radical anions in close proximity along the $a$ axis. Couplings $J_{a}$, and $J_{b}$ are depicted. d) Lowest unoccupied molecular orbital (LUMO) of neutral pterphenyl, showing bonding interactions between phenyl rings and along the long axis of the molecule. e) Carbon assignment of p-terphenyl.

Results and Discussion: Superconducting materials from doped organics are typically formed by doping a powder, solid-state crystal, or polycrystalline film in the solid state through sublimation of the material. ${ }^{20-22}$ However, this method leads to competition between intercalation and decomposition as shown for other PAHs. ${ }^{23}$ We find that be heating $p$-terphenyl with potassium metal under vacuum at $12-170{ }^{\circ} \mathrm{C}$ for $24-48 \mathrm{~h}$, results in a rearrangement and redistribution of the three aromatic rings that make up $p$-terphenyl (see the Supporting Information). To avoid this issue, we dope $p$ terphenyl with $\mathrm{K}$ using a solution-based approach, which was recently employed by Rosseinsky et al. for the alkali-doping of phenanthrene. ${ }^{14}$ The key is the use of [2.2.2] cryptand (222) to bind the $\mathrm{K}^{+}$ion (Figure $1 \mathrm{a}$ ). Figure $1 \mathrm{~b}$ and $1 \mathrm{c}$ display the structure of $[\mathrm{K}(\mathbf{2 2 2})]_{2}\left[p\right.$-terphenyl $\left.{ }_{3}\right]$, as determined by single crystal $\mathrm{X}$-ray diffraction (SCXRD). Inspecting the unit cell, we see that the structure 
contains two unique $p$-terphenyls: one shown in black and the other two (equivalent) shown in blue. Based on the structural parameters of the two types of $p$-terphenyl, we conclude that the blue molecules are anionic while the black ones are neutral. The average inter-phenyl bond length for the blue $p$-terphenyl is substantially shorter with values of $1.451 \AA$ (Figure 1e: 4-7 and 10-13), compared to the average $1.484 \AA$ for the black molecule. These distances are consistent with the semiquinoidal structure of the reduced $p$-terphenyl. ${ }^{19}$ The other $\mathrm{C}-\mathrm{C}$ bond lengths lead to the same conclusion, although, as expected, the variations are smaller: the average $\mathrm{C}-\mathrm{C}$ bonds parallel to the long axis (Figure 1e: 2-3; 5 6 ; $8-9 ; 11-12 ; 14-15 ; 17-18)$ are shorter $(1.379 \AA$ vs. $1.386 \AA)$ whereas the average value of the other $\mathrm{C}-\mathrm{C}$ bonds is larger $(1.409$ $\AA$ vs. $1.396 \AA$ ) for the blue $p$-terphenyls. These observations are consistent with population of the LUMO (Figure 1d) in the blue $p$ terphenyls while the black $p$-terphenyls remain thus formally neutral.

The anion of $p$-terphenyl is well known. Gas-phase spectroscopy has shown $p$-terphenyl to have an electron affinity (EA) of $0.4 \mathrm{eV}$. While this implies that $p$-terphenyl can hold some charge, it does call into question the kinetic stability of the anionic $p$-terphenyl. However, in the solid the anions are stabilized by the electrostatic field of the cations. The simple interpretation that there are two $p$ terphenyl radical anions and one neutral $p$-terphenyl is in fact an oversimplification. Our magnetic and theoretical studies described below show that the system is much more complex due to the low electron affinity of $p$-terphenyl.

We investigated the magnetic properties of $[\mathrm{K}(\mathbf{2 2 2})]_{2}[p$ terphenyl ${ }_{3}$ ] using magnetic susceptibility and electron spin resonance (ESR) measurements. Figure 2a displays the magnetic susceptibility (measured by SQUID magnetometry). The magnetic susceptibility increases with decreasing temperature, reaching a maximum near $25 \mathrm{~K}$ and a subsequent upturn below $10 \mathrm{~K}$. Above $70 \mathrm{~K}$, the susceptibility follows the Curie-Weiss law, $\chi(T)=$ $\frac{C}{T-\Theta_{C W}}$, where $C$ is the Curie constant, and the $\theta_{C W}$ Weiss constant, which gives an estimate of the local magnetic interactions. A fit to the high temperature data yields $\theta_{C W}=-60 \mathrm{~K}$ indicating antiferromagnetic interactions. The effective magnetic moment is calculated to be $\sim 0.4 \mu_{\mathrm{B}}$ per terphenyl at room temperature (See Supplementary Information). As presented below, the Néel temperature was found to be $\mathrm{T}_{N}=13 \mathrm{~K}$ from ESR data. The upturn below $10 \mathrm{~K}$ may reveal a minute contribution $(<2 \%)$ of paramagnetic impurities (see Supplementary Information Figure S6). The maximum at $25 \mathrm{~K}$, slightly below the Curie-Weiss temperature, is characteristic of strongly antiferromagnetically (AFM) coupled, low-dimensional systems (Figure 2a). ${ }^{24}$

ESR measurements on a single crystal of $[\mathrm{K}(\mathbf{2 2 2})]_{2}\left[p\right.$-terphenyl $\left.{ }_{3}\right]$ exhibit a single narrow $(\sim 0.08 \mathrm{mT}$ broad) Lorentzian ESR line above $20 \mathrm{~K}$ (see Figure $2 b$ inset). This narrow ESR signal is typical of exchange-narrowed low-dimensional organic systems. ${ }^{24-26}$

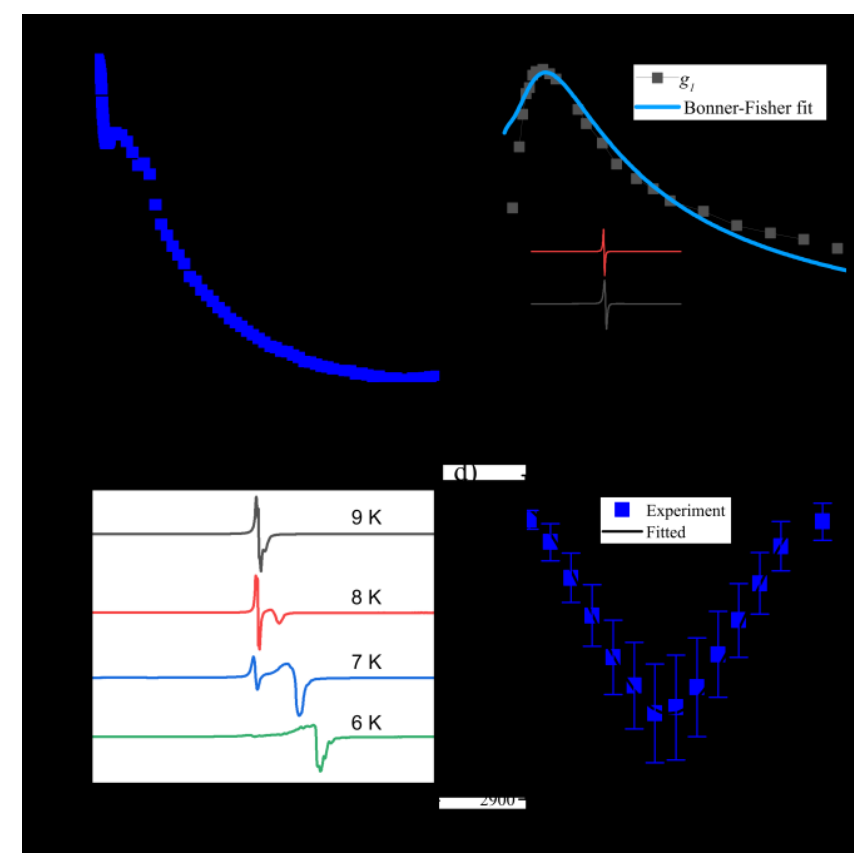

Figure 2 a) Magnetic susceptibility (FC) measured by SQUID magnetometry at $1 \mathrm{~T}$. The observed feature at $50 \mathrm{~K}$ in our SQUID data stems from impurity. b) Spin-susceptibility of $[\mathrm{K}(\mathbf{2 2 2})]_{2}[\mathrm{p}$ terphenyl ${ }_{3}$ ] measured by ESR, with $\mathrm{g}_{1}$ as the dimensionless magnetic moment. Solid line is a fit to the Bonner-Fisher model of spin$1 / 2$ chains. The inset shows individual ESR spectra of a $[\mathrm{K}(\mathbf{2 2 2})]_{2}\left[\mathrm{p}\right.$-terphenyl $\left.{ }_{3}\right]$ single crystal. c) Temperature evolution of the ESR spectra in the AFM state in the B $\mid \mathrm{b}$ direction at 9.4 $\mathrm{GHz}$. The vanishing ESR signal around $\mathrm{B}=3350 \mathrm{G}(\mathrm{g}=2)$ originates from the paramagnetic state. The broad line emerging at higher fields is an AFMR mode stemming from the ordered state. The shift of the AFMR mode (compared to the $\mathrm{g}=2$ paramagnetic signal) decreases with temperature. d) Angle dependence of the AFMR mode at $9.4 \mathrm{GHz}$, with the magnetic field, rotated in the b-c plane at $5 \mathrm{~K}$. Solid line is a fit to an easy-intermediate rotation of an AFMR mode with $\Omega_{=}=3 \mathrm{GHz}$ (and $\Omega_{+}>50 \mathrm{GHz}$ ). The error bars of the points indicate the linewidth of the AMR signals.

Temperature-dependent ESR measurements were performed on a single crystal of $[\mathrm{K}(\mathbf{2 2 2})]_{2}\left[p\right.$-terphenyl $\left.{ }_{3}\right]$ to track the spinsusceptibility of the spin component (Figure $2 \mathrm{~b}$ ). It shows a broad maximum around $25 \mathrm{~K}$, in good agreement with the SQUID magnetic susceptibility data. Below $20 \mathrm{~K}$, we observe an abrupt drop of the paramagnetic ESR signal intensity (Figure $2 \mathrm{~b}$ ). ${ }^{27}$ Along with the drop of the susceptibility, the ESR signal in the inset shifts to lower fields (by 0.1-0.2 mT) and broadens slightly. We attribute this to a paramagnetic-antiferromagnetic phase transition. These changes reveal strong antiferromagnetic fluctuations in our system. Interestingly, these emerge only a few $\mathrm{K}$ above the magnetic order, similarly to previously studied antiferromagnets in other lowdimensional organic systems. Specifically, the temperature dependence of the susceptibility resembles that of quasi-one-dimensional spin chain systems and points to the presence of weak spin anisotropy. This temperature dependence of the susceptibility is described by the Bonner-Fisher model of one-dimensional spin- $1 / 2$ chains to fit the ESR spin-susceptibility data above $20 \mathrm{~K}$, shown in blue in Figure $2 \mathrm{~b} .^{28}$ This analysis yields an estimate of dominant, intra-chain coupling of $40 \mathrm{~K}$ (defined as the coupling corrected by 
$k_{B}$, the Boltzmann constant); we define this coupling as simply $J_{x}(x$ $=a, b$, or $c$ ) in the remainder of the text. As shown in Figure $2 c$, the paramagnetic ESR signal gradually vanishes with temperature below $15 \mathrm{~K}$. At the same time, a new, broad ESR line emerges. This new low-temperature signal is substantially shifted to higher field compared to the paramagnetic ESR signal. Below the antiferromagnetic transition temperature, the spins contributing to the paramagnetic signal mixes into the antiferromagnetic phase and give signals at a different field. This new signal is assigned to an antiferromagnetic resonance (AFMR) mode of $[K(\mathbf{2 2 2})]_{2}[p$ terphenyl $\mathrm{l}_{3}$, which is a unique signature -only observed in pure systems- and it captures the spin dynamics of the antiferromagnetic phase. $^{29}$

Through angle-dependent measurements, one can identify the magnetic easy, intermediate and hard axes in an antiferromagnet. The angle- and field-dependence of the AFMR modes were fitted using a simulation based on the classical mean-field model of Nagamiya and Yosida. ${ }^{30}$ The simulation takes the ESR experimental frequency and the resonant field positions as input, and it calculates the deviation of the experimental and theoretical resonant field positions as a function of the easy-intermediate anisotropy energy $\left(\mathrm{K}_{1}\right)$. The resulting fit is presented in Figure $2 \mathrm{~d}$. This yields $\Omega_{-}=3 \mathrm{GHz}$, which is the measure of the anisotropy in frequency. Figure $2 \mathrm{~d}$ depicts the angle dependence of the AFMR mode at $9.4 \mathrm{GHz}$ for magnetic field orientations in the $b$ - $c$ plane at $5 \mathrm{~K}$. We conclude that the closely sinusoidal angle dependence stem from an easy-intermediate rotation in the plane. Otherwise, the resonant field position would exhibit a divergent behavior. These AFMR modes, combined with our high-frequency ESR studies (see Supplementary Information) unambiguously show that the AFM state exhibits triaxial anisotropy in $[\mathrm{K}(\mathbf{2 2 2})]_{2}[p$ terphenyl $\left.{ }_{3}\right]$, meaning that all three axes are magnetically different. In our model, we assign the crystallographic a direction to the hard axis, $c$ to the intermediate, and $b$ to the easy axis.

Spin-polarized DFT calculations (see details in the SI) were carried out for different ferromagnetic (FM) and AFM spin configurations of the full lattice. The full AFM configuration (Figure S1) was found to be the most stable spin configuration. These calculations reveal that the negative charge is confined into the subset of blue $p$ terphenyls. The results of these calculations were indistinguishable from those based only on the blue $p$-terphenyls sublattice and a background uniform charge, which we used to explore the possible role of disorder (see Supplementary Information). The strongest coupling $\left(J_{\mathrm{a}}\right)$ was calculated to be $+96 \mathrm{~K}$ (where the positive sign refers to AFM interactions) and is along the $1 \mathrm{D} p$-terphenyl chains. This value is in fair agreement with the value of $+40 \mathrm{~K}$, obtained, as discussed above, from a fit of the experimental data to a simple quasi-one-dimensional spin chain model. The different zigzag chains along $a$ are coupled through interaction $J_{\mathrm{b}}$ leading to a twodimensional $(a b)$ network of $J_{\mathrm{a}}$ and $J_{\mathrm{b}}$ magnetic interactions and these planes can couple through $J_{c}$ (Figure $1 \mathrm{~b}$ ). The calculated values for $J_{\mathrm{b}}$ and $J_{\mathrm{c}}$ are $+12 \mathrm{~K}$ and $+36 \mathrm{~K}$, respectively. Consequently, even if, as usual with Perdew-Burke-Ernzerhof (PBE)-type calculations, the magnetic interactions are somewhat larger than the experimental ones (see also Figure S2), this study suggests relatively strong AFM interactions along $a$, weaker AFM interactions along $c$ and very weak AFM interactions along $b$, in reasonable agreement with the hard, easy and intermediate axes assigned with ESR measurements.

The shortest C...C distance associated with interactions $J_{c}$ is $4.4 \AA$ (compared to 4.1-4.2 $\AA$ for $J_{\mathrm{b}}$ ), yet $J_{\mathrm{c}}$ has a larger coupling than $J_{\mathrm{b}}$. This initially surprising result can be explained through the LUMO...LUMO interactions (Figure $3 \mathrm{a}$ ). The C $2 \mathrm{p}_{\mathrm{z}}$ orbitals in $J_{c}$ are pointed angularly towards each other, creating more $\sigma$ character than in the parallel configured $J_{b}$, consequently creating a better overlap in $J_{c}$ than in the parallel configured $J_{b}$. Since the $J$ coupling constants are roughly proportional to the square of the overlaps, $J_{c}$ should be stronger than $J_{b}$. An estimation of the LUMO...LUMO overlap integrals associated with the three magnetic coupling interactions leads to a ratio $\left(\mathrm{S}_{\mathrm{a}}\right)^{2} /\left(\mathrm{S}_{\mathrm{b}}\right)^{2} /\left(\mathrm{S}_{\mathrm{c}}\right)^{2}$ of 5.3/1.0/1.6 in qualitative agreement with the ratio of DFT magnetic coupling constants $J_{\mathrm{a}} / J_{\mathrm{b}} / J_{\mathcal{O}} 8 / 1 / 3$. Thus, the conjugated chains of $p$-terphenyls are mainly coupled through $J_{\mathcal{O}}$, with a small coupling along $J_{\mathrm{b}}$.

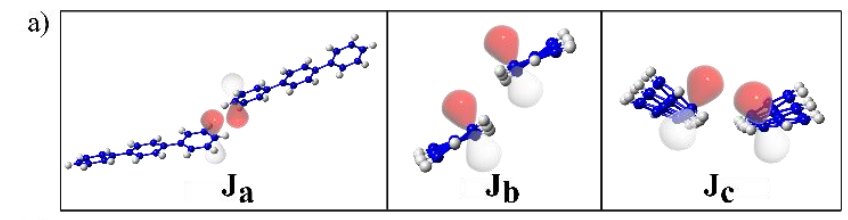

b)

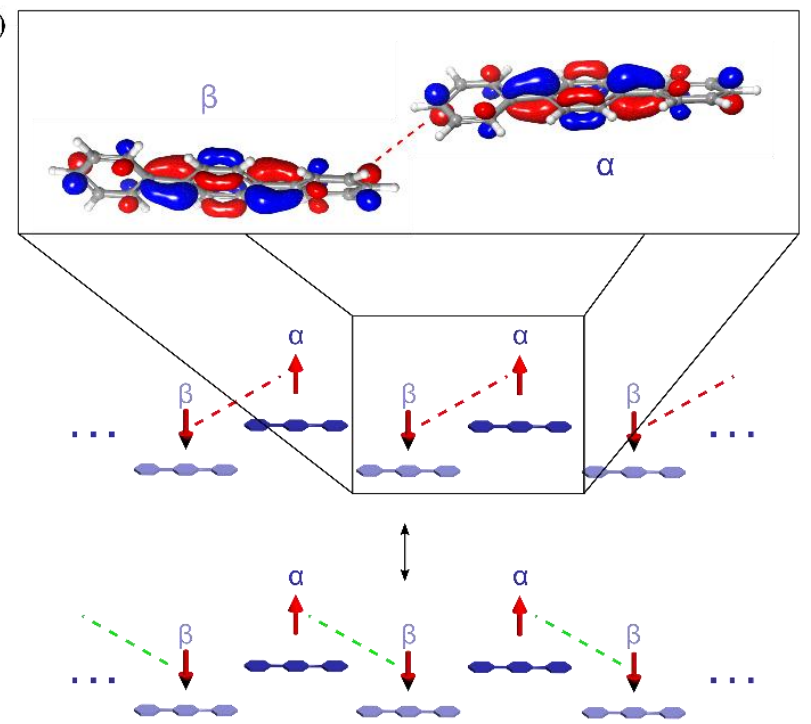

Figure 3 a) Schematic representation of the three different interactions $J_{a}>J_{c}>J_{b}$. Shown are the orbitals providing mostly $\sigma$-type orbital overlap in $J_{a}$; parallel in $J_{b}$; and angular towards each other in $J_{c}$. Orbitals pictured are simplified representations of the actual orbital shown in Figure 1d. b) Schematic representation of the $\mathrm{J}_{\mathrm{a}}$ with two possible resonance structures: $\alpha-\beta$ electrons interacting on either side of a molecule through red or green coupling. The delocalization of the coupling resembles conjugated systems such as polyacetylene or benzene, but rather on a molecular scale introducing intermolecular resonance. Inset shows the red $\alpha-\beta$ coupling.

From a structure/property perspective $\left.[\mathrm{K}(\mathbf{2 2 2})]_{2}[p \text {-terphenyl }]_{3}\right]$ exhibits remarkable features with the occurrence of both charged and neutral $p$-terphenyls, as well as chains of $p$-terphenyl radical anions that couple antiferromagnetically. As shown by DFT calculations, the two $p$-terphenyl anions of a $([\mathrm{K}(\mathbf{2 2 2})] \text { - } p \text {-terphenyl })_{2}$ dimeric unit will tend to couple along the $a$ direction. This leads to a $\left.[\text { trans-( } p \text {-terphenyl })_{2}\right]^{2-}$ dimer through a somewhat loose $\mathrm{C} 1-$ $\mathrm{C} 16$ bond, i.e., the two "anionic" electrons -one on each terphenyl 
anion- interact and are low-spin coupled. In such unit, every $p$ terphenyl anion faces a $([\mathrm{K}(\mathbf{2 2 2})]$ fragment. Apparently these isolated $([\mathrm{K}(\mathbf{2 2 2})] \text {-p-terphenyl })_{2}$ dimers are not able to fill space in a compact manner, and thus construct a periodic, organic solid having such a high density of localized charge. In order to reach a $1: 1$ ratio of $[\mathrm{K}(\mathbf{2 2 2})]$ and $p$-terphenyl anions, the system utilizes the electronically "innocent" as structurally and chemically compatible filler. The neutral $p$-terphenyl fits these requirements. Because of their shape, these neutral molecules are easily adaptable. Moreover, these neutral $p$-terphenyls do not have extra charges that can disturb the packing, and they also have many outer $\mathrm{C}-\mathrm{H}$ bonds which can make weak hydrogen bonding interactions that hold the solid together. Under such conditions, the $p$-terphenyl anions find their way to extend into weakly coupled chains. The extra, "anionic", electrons form weak bonds between adjacent anions, but rather than each $p$-terphenyl anion forming just a single bond to one of its neighbours, each forms a bond to both of its neighbours along the chain. This seemingly impossible hypervalent situation is the result of valence-bond resonance (as is seen in benzene at the molecular scale, and polyacetylene at the extended scale) in which two or more related but distinct bonding patterns constructively interfere (See Figure 3b). It should be emphasized that the resonance structure in our system is intermolecular, rather than intramolecular. Furthermore, note that these antiferromagnetically coupled chains can even gain some additional weak stabilization by antiferromagnetically coupling along the inter-chain directions. This stabilization is the subtle mechanism behind the structure and magnetic properties of $[\mathrm{K}(\mathbf{2 2 2})]_{2}\left[p\right.$-terphenyl $\left.{ }_{3}\right]$.

Conclusion: We have synthesized and magnetically characterized phase-pure molecular solids consisting of $p$-terphenyl, $\mathrm{K}$, and a chelating agent. The (222) cryptand provides control over the crystallization process, resulting in materials with well-defined stoichiometries. Subsequently, we find that in $[\mathrm{K}(\mathbf{2 2 2})]_{2}[p$ terphenyl ${ }_{3}$ ] neutral $p$-terphenyl molecules are interspersed between uniform chains of $p$-terphenyl radical anions, each bearing a $1 / 2$ spin. An AFM phase sets in at $13 \mathrm{~K}$. The observed AFMR (present only in pure systems) provides a clear-cut signature of the threedimensional magnetic order, and allows the exact determination of the exchange anisotropies. The dominant intrachain antiferromagnetic exchange interaction of $40 \mathrm{~K}$ occurs between neighboring $p$ terphenyls in the chains that run along the $a$ direction. This antiferromagnetic insulator may host high $\mathrm{T}_{\mathrm{c}}$ superconductivity as in the case of hole doped cuprates. It implies that the high $\mathrm{T}_{c}$ reported for a polycrystalline K-doped $p$-terphenyl might be a such minority phase. ${ }^{31}$ It would be extremely interesting for our high quality single crystal to explore the phase diagram for both electron and hole doping, with the goal of observing superconductivity, in a field effect transistor configuration. On the chemistry side, our approach permits the rational design of other original low-dimensional alkalidoped organic systems by varying the chelating agents, alkali-, alkaline-earth metals, or even by combining two different encapsulated dopants for controlling the stoichiometry. ${ }^{32}$

\section{ASSOCIATED CONTENT}

Supporting Information. Experimental details, ESR explanation, DFT calculations, ESR, SQUID, crystallographic data. This material is available free of charge via the Internet at http://pubs.acs.org.

\section{AUTHOR INFORMATION}

\section{Corresponding Authors}

* Patrick Batail, patrick.batail@univ-angers.fr

*Enric Canadell, canadell@icmab.es

* Michael L. Steigerwald, mls2064@columbia.edu

*Colin Nuckolls, cn37@columbia.edu

\section{Author Contributions}

The manuscript was written through contributions of all authors. / All authors have given approval to the final version of the manuscript.

\section{ACKNOWLEDGMENT}

C.N. thanks Sheldon and Dorothea Buckler for their generous support. Support for this research was provided by the Center for Precision Assembly of Superstratic and Superatomic Solids, an NSF MRSEC (award number DMR-1420634), and the Air Force Office of Scientific Research (award number FA9550-18-1-0020). R.H.S. acknowledges the support from the Columbia Nano Initiative Postdoctoral Fellowship. X.R. acknowledges support from the NSF CAREER award (NSF DMR-1751949). Single crystal X-ray diffraction was performed at the Shared Materials Characterization Laboratory at Columbia University, maintained using funding from Columbia University for which we are grateful. Work in Lausanne was supported by the Swiss National Science Foundation.

Work in Spain was supported by MICIU (Spain) through Grants PGC2018-096955-B-C44 and PGC2018-093863-B-C22, Generalitat de Catalunya (2017SGR1506 and 2017SGR1289). E C acknowledges support of the Spanish MINECO through the Severo Ochoa Centers of Excellence Program (SEV-2015-0496) and P A from the Maria de Maeztu Units of Excellence Program (MDM-2017-0767). S C was supported by FONDECYT (Chile) through project 11107163.

\section{ABBREVIATIONS}

(222), [2.2.2.]Cryptand; AFM, antiferromagnetic; DFT, density functional theory; $\mathrm{PAH}$, polycyclic aromatic hydrocarbon; EA, electron affinity; LUMO, lowest unoccupied molecular orbital; ESR, electron spin resonance; SQUID, superconducting quantum interference device; AFMR, antiferromagnetic resonance; $\mathrm{ZFC}$, zero-field cool; FC, field cool; PBE, Perdew-Burke-Ernzerhof

\section{REFERENCES}

(1) Romero, F. D.; Pitcher, M. J.; Hiley, C. I.; Whitehead, G. F. S.; Kar, S.; Ganin, A. Y.; Antypov, D.; Collins, C.; Dyer, M. S.; Klupp, G.; Colman, R. H.; Prassides, K.; Rosseinsky, M. J. Redox-Controlled Potassium Intercalation into Two Polyaromatic Hydrocarbon Solids. Nat. Chem. 2017,9 (7), 644-652. https://doi.org/10.1038/nchem.2765.

(2) Hebard, A. F.; Rosseinsky, M. J.; Haddon, R. C.; Murphy, D. W.; Glarum, S. H.; Palstra, T. T. M.; Ramirez, A. P.; Kortan, A. R. Superconductivity at $18 \mathrm{~K}$ in Potassium-Doped C60. Nature 1991, 350 (6319), 600-601. https://doi.org/10.1038/350600a0.

(3) Ganin, A. Y.; Takabayashi, Y.; Khimyak, Y. Z.; Margadonna, S.; Tamai, A.; Rosseinsky, M. J.; Prassides, K. Bulk Superconductivity at $38 \mathrm{~K}$ in a Molecular System. Nat. Mater. 2008, 7 (5), 367-371. https://doi.org/10.1038/nmat2179.

(4) Saito, G.; Yoshida, Y. Organic Superconductors. Chem. Rec. 2011, 11 (3), 124-145. https://doi.org/10.1002/tcr.201000039.

(5) Ganin, A. Y.; Takabayashi, Y.j Jeglič, P.; Arčon, D.; Potočnik, A.; Baker, P. J.; Ohishi, Y.; McDonald, M. T.; Tzirakis, M. D.; McLennan, A.; Darling, G. R.; Takata, M.; Rosseinsky, M. J.; Prassides, K. Polymorphism 
Control of Superconductivity and Magnetism in Cs3 C60 Close to the Mott Transition. Nature 2010, 466 (7303), 221-225. https://doi.org/10.1038/nature09120.

(6) Craciun, M. F.; Giovannetti, G.; Rogge, S.; Brocks, G.; Morpurgo, A. F.; Van Den Brink, J. Evidence for the Formation of a Mott State in Potassium-Intercalated Pentacene. Phys. Rev. B - Condens. Matter $\begin{array}{lllll}\text { Mater. } & \text { Phys. 2009, } & 79 & \text { (12), } & 125116 .\end{array}$ https://doi.org/10.1103/PhysRevB.79.125116.

(7) Liu, W.; Lin, H.; Kang, R.; Zhu, X.; Zhang, Y.; Zheng, S.; Wen, H. H. Magnetization of Potassium-Doped $\mathrm{p}$-Terphenyl and $\mathrm{p}$ Quaterphenyl by High-Pressure Synthesis. Phys. Rev. B 2017, 96 (22), 224501.https://doi.org/10.1103/PhysRevB.96.224501.

(8) Wang, X. F.; Liu, R. H.; Gui, Z.; Xie, Y. L.; Yan, Y. J.; Ying, J. J.; Luo, X. G.; Chen, X. H. Superconductivity at $5 \mathrm{~K}$ in Alkali-Metal-Doped Phenanthrene. 2011. https://doi.org/10.1038/ncomms1513.

(9) Mitsuhashi, R.; Suzuki, Y.; Yamanari, Y.; Mitamura, H.; Kambe, T.; Ikeda, N.; Okamoto, H.; Fujiwara, A.; Yamaji, M.; Kawasaki, N.; Maniwa, Y.; Kubozono, Y. Superconductivity in Alkali-Metal-Doped Picene. Nature 2010, 464. https://doi.org/10.1038/nature08859.

(10) K. Tanigaki, T. W. Ebbensen, S. Saito, J. Mizuki, J. S. Tsai, Y. K. \& S. K. Superconductivity at $33 \mathrm{~K}$ in Cs, Rb, C60. Lett. to Nat. 1991, 352, 222-223.

(11) Xue, M.; Cao, T.; Wang, D.; Wu, Y.; Yang, H.; Dong, X.; He, J.; Li, F.; Chen, G. F. Superconductivity above $30 \mathrm{~K}$ in Alkali-Metal-Doped Hydrocarbon. https://doi.org/10.1038/srep00389.

(12) Wang, R.-S.; Gao, Y.; Huang, Z.-B.; Chen, X.-J. Superconductivity above 120 Kelvin in a Chain Link Molecule. 2017, 1-19.

(13) Kosugi, T.; Miyake, T.; Ishibashi, S.; Arita, R.; Aoki, H. FirstPrinciples Electronic Structure of Solid Picene. https://doi.org/10.1143/JPSJ.78.113704.

(14) Takabayashi, Y.; Menelaou, M.; Tamura, H.; Takemori, N.; Koretsune, T.; Štefančič, A.; Klupp, G.; Buurma, A. J. C.; Nomura, Y.; Arita, R.; Arčon, D.; Rosseinsky, M. J.; Prassides, K. $\pi$-Electron $S=1 / 2$ Quantum Spin-Liquid State in an Ionic Polyaromatic Hydrocarbon. Nat. Chem. 2017, 9 (7), 635-643. https://doi.org/10.1038/nchem.2764.

(15) Heguri, S.; Kobayashi, M.; Tanigaki, K. Questioning the Existence of Superconducting Potassium Doped Phases for Aromatic Hydrocarbons. Phys. Rev. B - Condens. Matter Mater. Phys. 2015, 92 (1), 014502. https://doi.org/10.1103/PhysRevB.92.014502.

(16) Rosseinsky, M. J.; Murphy, D. W.; Fleming, R. M.; Tycko, R.; Ramirez, A. P.; Dabbagh, G.; Barrett, S. E. Structural and Electronic Properties of Sodium-Intercalated C60. Nature 1992, 356 (6368), 416-418. https://doi.org/10.1038/356416a0.

(17) Park, K. Quantum Antiferromagnetism and High Tc Superconductivity: A Close Connection between the t-J Model and the Projected BCS Hamiltonian. Phys. Rev. B - Condens. Matter Mater. Phys. 2005, 72 (24), 245116. https://doi.org/10.1103/PhysRevB.72.245116.

(18) Bourbonnais, C.; Sedeki, A. Link between Antiferromagnetism and Superconductivity Probed by Nuclear Spin Relaxation in Organic Conductors. Phys. Rev. B - Condens. Matter Mater. Phys. 2009, 80 (8), 085105. https://doi.org/10.1103/PhysRevB.80.085105.
(19) Takabayashi, Y.; Ganin, A. Y.; Jeglič, P.; Arčon, D.; Takano, T.; Iwasa, Y.; Ohishi, Y.; Takata, M.; Takeshita, N.; Prassides, K.; Rosseinsky, M. J. The Disorder-Free Non-BCS Superconductor CS3C60 Emerges from an Antiferromagnetic Insulator Parent State. Science (80- ). 2009, 323 (5921), 1585-1590. https://doi.org/10.1126/science.1169163.

(20) Wang, P.; Metzger, R. M.; Bandow, S.; Maruyama, Y. Superconductivity in Langmuir-Blodgett Multilayers of C60 Doped with Potassium. J. Phys. Chem. 1993, 97 (12), 2926-2927. https://doi.org/10.1021/j100114a016.

(21) Miura, Y. F.; Horikiri, M.; Saito, S. H.; Sugi, M. Evidence for Superconductivity in Langmuir-Blodgett Films of Ditetradecyldimethylammonium-Au(Dmit)2. Solid State Commun. 2000, 113 (11), 603-605. https://doi.org/10.1016/S0038-1098(99)00559-1.

(22) Kubozono, Y.; Mitamura, H.; Lee, X.; He, X.; Yamanari, Y.; Takahashi, Y.; Suzuki, Y.; Kaji, Y.; Eguchi, R.; Akaike, K.; Kambe, T.; Okamoto, H.; Fujiwara, A.; Kato, T.; Kosugi, T.; Aoki, H. MetalIntercalated Aromatic Hydrocarbons: A New Class of Carbon-Based Superconductors. Phys. Chem. Chem. Phys. Phys. Chem. Chem. Phys 1647, 13 (13), 16476-16493. https://doi.org/10.1039/c1cp20961b.

(23) Zhang, J.; Whitehead, G. F. S.; Manning, T. D.; Stewart, D.; Hiley, C. I.; Pitcher, M. J.; Jansat, S.; Prassides, K.; Rosseinsky, M. J. Reactivity of Solid Rubrene with Potassium: Competition between Intercalation and Molecular Decomposition. J. Am. Chem. Soc. 2018, 140 (51), 18162 18172. https://doi.org/10.1021/jacs.8b11231.

(24) Torrance, J. B.; Tomkiewicz, Y.; Silverman, B. D. Enhancement of the Magnetic Susceptibility of TTF-TCNQ (TetrathiafulvaleneTetracyanoquinodimethane) by Coulomb Correlations. Phys. Rev. B 1977, 15 (10), 4738-4749. https://doi.org/10.1103/PhysRevB.15.4738.

(25) Náfrádi, B.; Olariu, A.; Forró, L.; Mézière, C.; Batail, P.; Jánossy, A. Spin Dynamics in the $S=1 / 2$ Antiferromagnetic Chain Compounds $\delta$ $\left.(\text { EDT-TTF-CONMe })_{2}\right)_{2} \mathrm{X}\left(\mathrm{X}=\mathrm{AsF}_{6}, \mathrm{Br}\right)$ : A Multi-Frequency Electron Spin Resonance Study. Phys. Rev. B 2010, 81 (22). https://doi.org/10.1103/physrevb.81.224438.

(26) Dumm, M.; Loidl, A.; Fravel, B. W.; Starkey, K. P.; Montgomery, L. K.; Dressel, M. Electron Spin Resonance Studies on the Organic LinearChain Compounds of quot;TMTCF... 2 X \& quot; CS,Se; XPF 6 ,AsF $6, \mathrm{ClO} 4$ ,Br....

(27) Zheng, W.; Singh, R. R. P.; Mckenzie, R. H.; Coldea, R. Temperature Dependence of the Magnetic Susceptibility for Triangular-Lattice Antiferromagnets with Spatially Anisotropic Exchange Constants. https://doi.org/10.1103/PhysRevB.71.134422.

(28) Bonner, J. C.; Fisher, M. E. Linear Magnetic Chains with Anisotropic Coupling. Phys. Rev. 1964, 135 (3A), A640.

(29) Coulon, C.; Clérac, R. Electron Spin Resonance: a major probe for molecular conductors. Chem. Rev. 2004, 104, 5655-5687.

(30) Nagamiya, T.; Yosida, K.; Kubo, R. Antiferromagnetism. Adv. Phys. 1955, 4 (13), 1-112. https://doi.org/10.1080/00018735500101154.

(31) Slezak J. A.; Jinho Lee, Wang M.; McElroy K.; Fujita K.; Andersen B. M.; Hirschfeld P. J.; Eisaki H.; Uchida S.; Davis J. C. PNAS 2008, 105 (9) 3203-3208.

(32) Mori, T. Organic Conductors with Unusual Band Fillings. Chem. Rev. 2004, 104 (11), 4947-4969. 
SYNOPSIS TOC

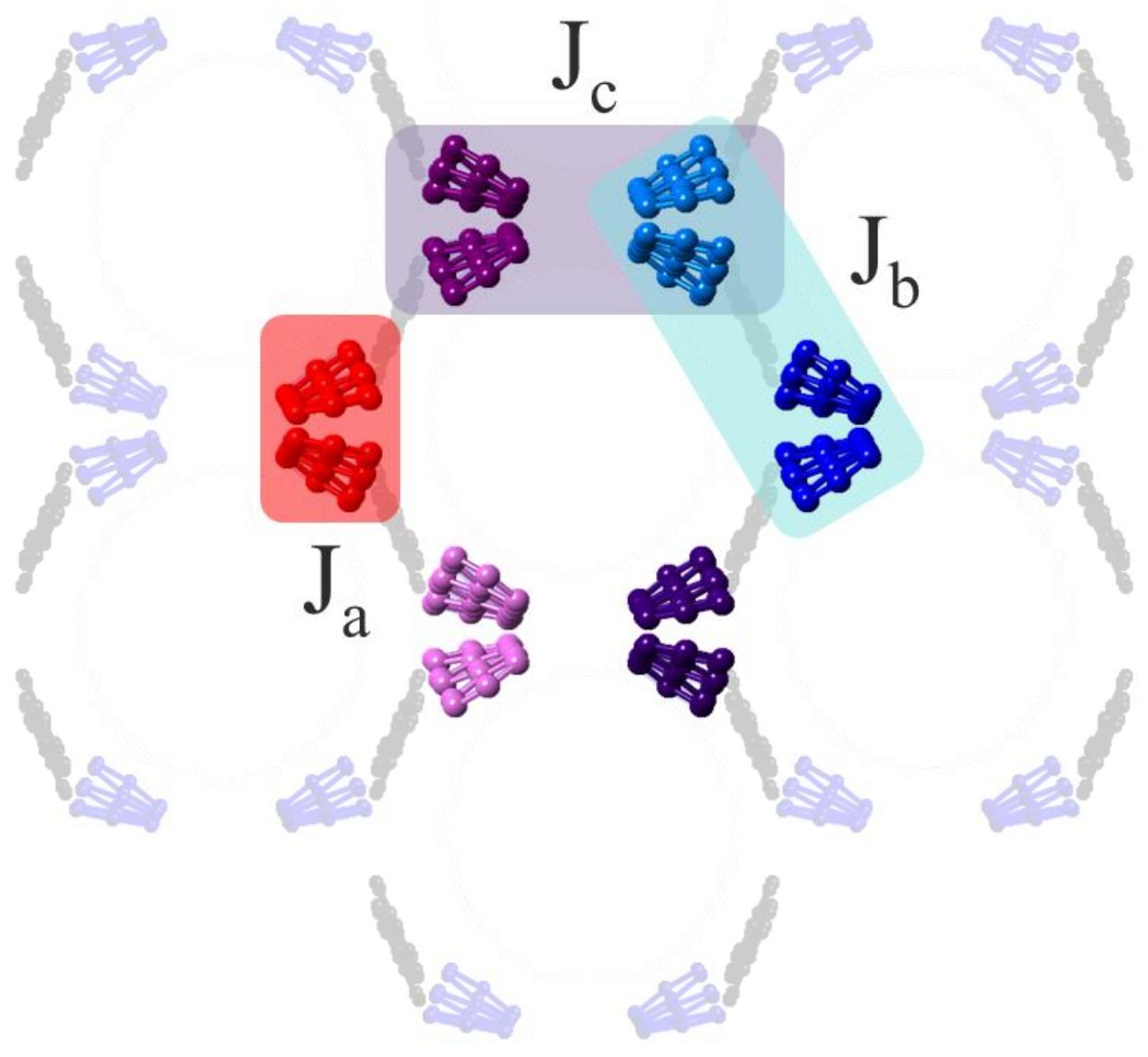

